\title{
Top Surface Imaging for Extreme Ultraviolet Lithography
}

\author{
Craig Henderson, David Wheeler, ${ }^{\ddagger}$ Timothy Pollagi, ${ }^{\ddagger}$ Greg Cardinale, \\ Donna O'Connell, Aaron Fisher, Veena Rao ${ }^{\dagger}$, and John Goldsmith \\ Sandia National Laboratories \\ PO Box 969, MS9405, Livermore, California 94551-0969 U.S.A. \\ ${ }^{\ddagger}$ PO Box 5800, MS1407, Albuquerque, New Mexico 87185-5800, U.S.A. \\ ${ }^{\dagger}$ Intel Corporation \\ MS SC1-03, 3065 Bowers Avenue, Santa Clara, California 95052 U.S.A.
}

\begin{abstract}
The use of extreme ultraviolet radiation (13.4 $\mathrm{nm}$ ) for microlithography requires a thin layer imaging resist technology due to the strongly absorbing nature of $13.4 \mathrm{~nm}$ radiation. In any thin layer imaging technique, the aerial image from the exposure system is transferred into a top imaging layer of photoresist and is used to form an in-situ mask for pattern transfering the image through the remaining resist thickness to the device layer in a separate etch development step. The work presented here takes advantage of a new aminodisilane silylation reagent to make improvements in the thin layer imaging process known as top surface imaging. This silylation process is capable of $100 \mathrm{~nm}$ resolution using extreme ultraviolet exposure at 13.4 $\mathrm{nm}$ and the new aminodisilane reagent.

Keywords: top surface imaging, dimethylaminodimethyldisilane, extreme ultraviolet lithography, thin layer imaging
\end{abstract}

\section{Introduction}

Thin layer imaging (TLI) involves the photochemical transfer of the aerial image into only the top portion of the photoresist in such a fashion that the image can be transferred through the rest of the resist thickness to the device layer in subsequent development steps [1-2]. Several possible TLI schemes are shown in Figure 1, where the illustrations show each process prior to the final pattern transfer step. In all of these processes, the pattern transfer is accomplished by using an etch process with a selectivity for materials containing refractory elements, such as silicon, or for nonrefractory materials. The schemes differ then in the method used to transfer the aerial image into a top surface pattern with only selected areas containing silicon or another refractory element.
In top surface imaging (TSI), also known as post-exposure silylation, either the unexposed or exposed regions can be selectively silylated by gaseous aminosilanes, depending on the chemistry of the photoresist [3-8]. In most cases, the diffusion and reaction of the silylation reagent is prevented in the exposed regions by photochemical crosslinking [35].

In bilayer, the entire top surface contains silicon prior to exposure. Either the exposed or unexposed regions are then removed by solution development after exposure, depending on whether the photochemistry makes the exposed resist more or less soluble in the chosen developer. A well-known variation of the TSI and bilayer process is the CARL process [9] where silicon is added to the top layer of a bilayer with a gas phase process, but only after the 

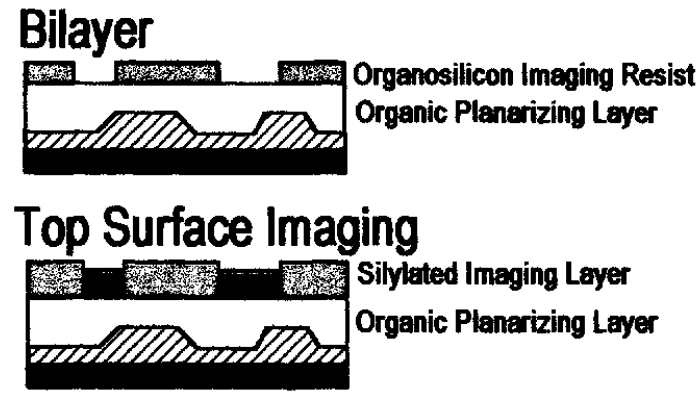

\section{SLR/Hard Mask or Trilayer}

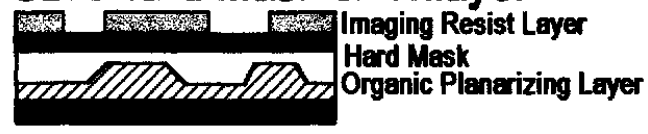

$\downarrow \begin{aligned} & \text { Dry Etch } \\ & \text { Development }\end{aligned}$

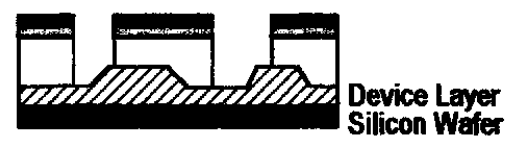

Fig. 1. Pattern transfer of the lithographic image to the device layer for several different possible TLI resist technologies.

top layer has been solution developed first. In all of these TLI processes involving organosilicon materials, the silicon in the top surface is converted to a silicon dioxide etch mask in the oxygen plasma etch, allowing pattern transfer of the image to the device layer (Figure 1).

The top, imaged layer does not have to contain silicon if the lower layer is a refractory hard mask, as shown in Figure 1 for the single layer resist over hard mask (SLR/hard mask) process. If the SLR/hard mask also includes an organic planarizing layer, the process is the more familiar trilayer process, where the three layers serve three separate functions of imaging, pattern transfer, and planarization.

Although many TLI processes were first described years ago, the success of the simpler solution-developed single layer resist (SLR) for optical lithography, including now $248 \mathrm{~nm}$ and 193 nm lithography, has meant that TLI has rarely been used for manufacturing. However, for extreme ultraviolet lithography (EUVL), a post-optical technology candidate, TLI is strictly required due to the highly attenuated nature of EUV radiation (13.4 $\mathrm{nm}$ ) in organic photoresists. The absorption of EUV radiation is atomistic in nature, and the necessary EUV absorption coefficients for a photoresist can be estimated simply from the empirical formula and density of the material [10]. For a common novolac resist, the effective working depth is only $70-100 \mathrm{~nm}$ [11], much thinner than the resist thickness generally required for device processing at the $100 \mathrm{~nm}$ design rule [12]. Thus, several TLI schemes are being evaluated in parallel as possible resist technologies for EUVL. This paper describes the evaluation of TSI for EUVL using the two 10x reduction EUV exposure tools at Sandia National Laboratories.

\section{Method}

A generalized process flow for TSI is as follows: silicon wafers were spin coated with a negative tone, chemically amplified photoresist to give approximately $400 \mathrm{~nm}$ of resist thickness. Following a post apply bake step, the wafers were patterned using $13.4 \mathrm{~nm}$ radiation in the $10 \mathrm{xI}$ exposure system (NA 0.06 or NA 0.07 ). The exposure dose was determined by comparison to the known exposure dose for a commercially available e-beam photoresist. The wafers were post-exposure baked to crosslink the photoresist in the exposed areas. Subsequent gas phase treatment of the wafers with the aminodisilane, dimethylaminodimethyldisilane (DMDS), in a single wafer silylation chamber silylated the wafers in the unexposed regions only, swelling the resist film and creating a latent image. Pattern transfer of this latent image to the silicon wafer was accomplished by a two-step dry etch process, including a short, non-selective breakthrough etch to remove the top most layer of resist across the entire surface, followed by a highly selective oxygen main etch. The oxygen etch transferred the pattern by anisotropically etching the exposed, non-silylated regions. This TSI process was positive tone overall.

All critical dimension (CD) and line edge roughness (LER) determinations were done with top down images from a field emission scanning electron microscope and an image analysis software application developed at Sandia National Laboratories. The line edge roughness values are given as a $3 \sigma$ deviation from the average line position along a single side of the printed line.

3. Results and Discussion

The majority of the TLI development work at EUV wavelength has been done on the TSI process, 


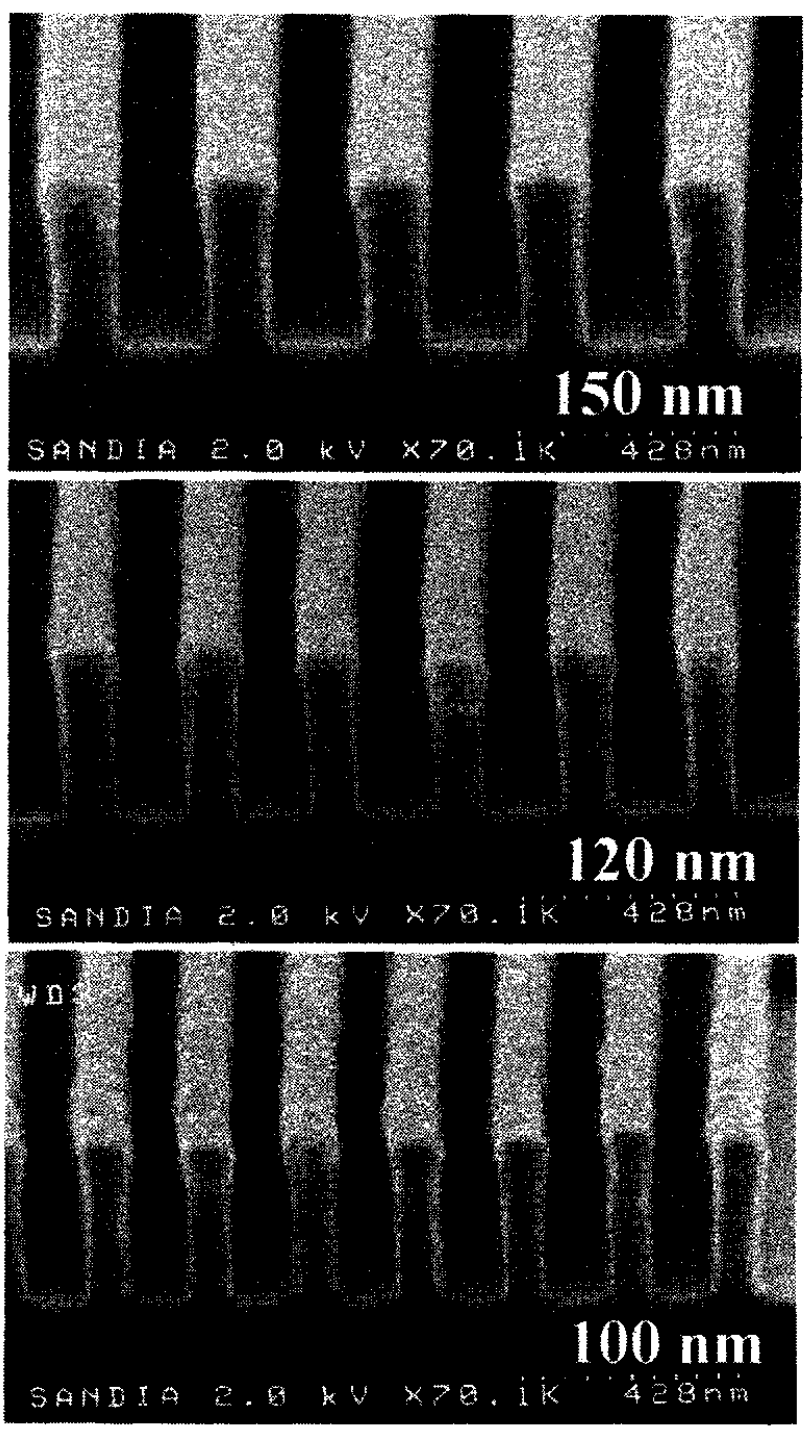

Fig. 2. Equal lines and spaces printed with $13.4 \mathrm{~nm}$ exposure on the 10xI system (NA 0.07) with an approximate dose of $13 \mathrm{~mJ} / \mathrm{cm} 2$ and TSI/DMDS processing.

mostly due to the availability of commercial photoresists suitable for TSI. In most cases, the resist used for TSI is a negative tone resist that crosslinks under exposure, thus preventing the diffusion of the silylation gas into these areas. Hydroxy aromatic polymers, such as novolacs and polyhydroxystyrene, are commonly chosen for TSI because these polymers are readily silylated by aminosilanes in the unexposed regions.
The high resolution possible with the TSI process is demonstrated for EUV lithography in Figure 2, which shows $100 \mathrm{~nm}$ resolution. These cross-sectioned equal lines and spaces were patterned in $400 \mathrm{~nm}$ of resist using $13.4 \mathrm{~nm}$ exposure (NA 0.07 ) and an approximate EUV dose of $13 \mathrm{~mJ} / \mathrm{cm}^{2}$. Dimethylaminodimethyldisilane (DMDS) was used as the silylation reagent.

The advantages of DMDS over other commonly used monosilanes include higher pattern transfer etch selectivity and higher effective photospeed [13]. The faster photospeed of the disilanes relative to monosilanes, such as dimethylsilyldimethylamine (DMSDEA), is assumed to be due to the larger size of the disilane reagent, thus requiring less dose and crosslinking to prevent diffusion. The better etch selectivity, which is attributed to the higher silicon content of the disilane reagent, has been measured at 45:1 for DMDS silylated photoresist relative to the unsilylated resist [13].

Silylation of hydroxy aromatic polymers generally decreases the glass transition temperature of the polymer and often results in the unwanted flow of resist, especially for larger features. Flow of silylated resist has been observed earlier for the disilane, dimethyl-aminopentamethyl-disilane (PMDS), and had to be controlled by the addition of a gaseous crosslinking reagent to the PMDS silylation gas [14]. The use of a crosslinker additive was not found to be necessary for the DMDS process, suggesting a smaller relative decrease in the glass transition temperature for the dimethyl reagent.

The thickness of the silicon etch mask decreases with smaller linewidth, eventually falling below the minimum thickness required to perform the pattern transfer etch without large $C D$ variations or complete loss of the etch mask [15]. The formation of an unwanted, thin layer of silylation in the exposed regions (Figure 3) necessitates the use of a nonselective "breakthrough" etch to remove this layer prior to the pattern transfer etch. Although necessary to prevent residue formation in the exposed, etched areas, the breakthrough etch clearly reduces the effective etch mask thickness and likely contributes to the resolution limit of the TSI process. Further material and process develop-ment is needed to prevent the unwanted surface silylation and, thus, improve the resolution and CD control of the TSI process for EUVL. 


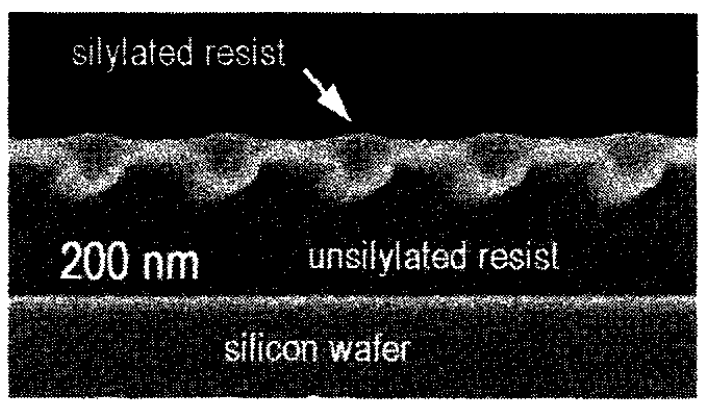

Fig. 3. Cross-sectional plasma stain of $200 \mathrm{~nm}$ equal lines and spaces after silylation and prior to normal etch development (193 nm exposure).

The top-down SEM images (Figure 4) of the same exposures shown in Figure 1 again demonstrate the $100 \mathrm{~nm}$ resolution possible for the TSI process and EUV exposure. These images can also be used to quantify the amount of line edge roughness (LER) produced by this particular TSI process using 13.4 $\mathrm{nm}$ exposure and silylation with DMDS. The amount of LER was measured for two different apertures (NA 0.06 and NA 0.07 ) of the same 10xI exposure system, and the results are given in Table 1. The $3 \sigma$ values given in Table 1 are for the deviation from the average line position along a single side of a resist line. Possible causes for the LER observed in this TSI process include silylation inhomogenities, resin inhomogenities and diluent effects, etch plasma effects, and mask roughness. Better understanding of the contributors to LER will be needed to reduce the magnitude of the LER closer to the values commonly observed for solution-developed optical lithography. The reduction in LER measured for the NA 0.07 aperture (Table 1) compared to the NA 0.06 aperture is thought to be due, at least in part, to a better aerial image for the larger aperture.

The degree of linearity, or ability of the lithographic process to print a variety of linewidths to within $\pm 5 \%$ of the coded linewidth in the same exposure field, was determined for the DMDS silylation process with EUV exposure. The results for dense lines (1:1 pitch) ranging from $200 \mathrm{~nm}$ to $100 \mathrm{~nm}$ in linewidth are shown in Figure 5 for two exposures with different numerical apertures. These results indicate that the TSI process with DMDS shows a reasonable amount of linearity $( \pm 5 \%$ of the coded linewidth) for linewidths from $200 \mathrm{~nm}$ to 100 nm printed with EUV exposure.

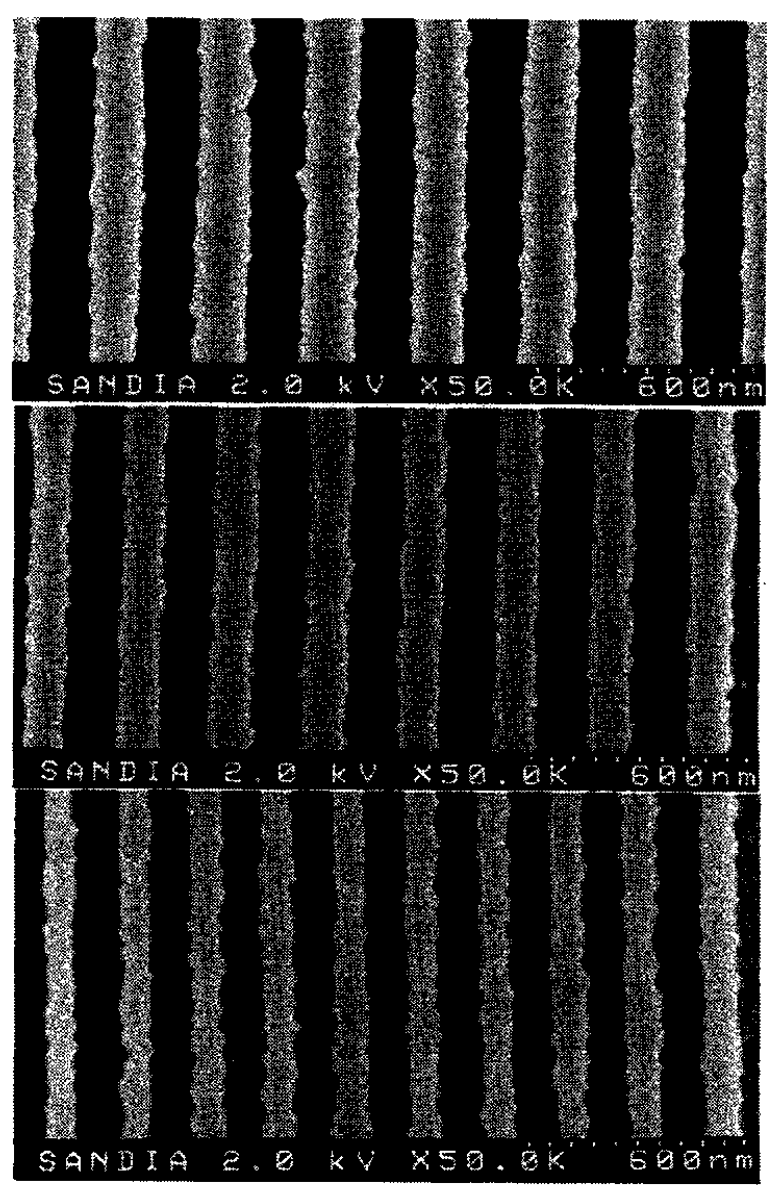

Fig. 4. Top down SEM images of equal lines and spaces (150 nm linewidth top, $130 \mathrm{~nm}$ linewidth middle, and $100 \mathrm{~nm}$ linewidth bottom) in $400 \mathrm{~nm}$ of resist printed with EUV lithography and TSI processing.

\begin{tabular}{|c|c|c|}
\hline \multirow[b]{2}{*}{$\begin{array}{l}\text { Linewidth } \\
\text { (nm) }\end{array}$} & \multicolumn{2}{|c|}{ Exposure at $13.4 \mathrm{~nm}$} \\
\hline & $\begin{array}{c}\text { NA } 0.07 \\
\text { LER (nm, 3 } 3 \sigma)\end{array}$ & $\begin{array}{c}\text { NA } 0.06 \\
\text { LER }(\mathrm{nm}, 3 \sigma)\end{array}$ \\
\hline 200 & 11.2 & 10.3 \\
\hline 175 & 12.2 & 10.6 \\
\hline 150 & 12.3 & 14.8 \\
\hline 130 & 11.4 & 16.4 \\
\hline 120 & 10.8 & 17.5 \\
\hline 100 & 12.1 & $>30$ \\
\hline
\end{tabular}

Table 1. Line edge roughness measurements ( $3 \sigma$, one side) for linewidths between $200-100 \mathrm{~nm}$ (1:1 pitch) for EUV exposure (NA 0.06 and NA 0.07) and DMDS/TSI processing. 


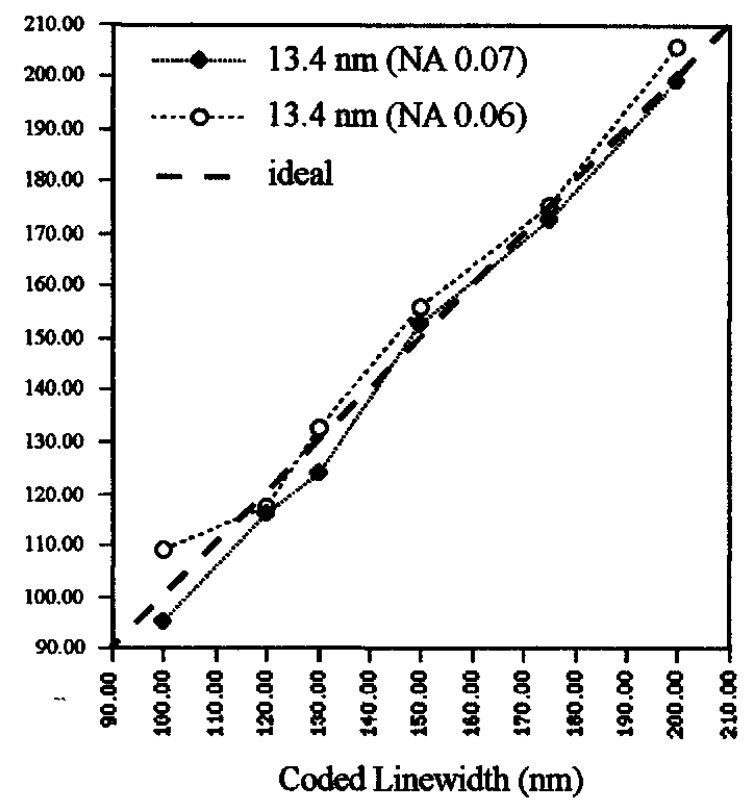

Fig. 5. Linearity plot of printed linewidth (nm) versus coded linewidth on mask for 10xI EUV exposure system (NA 0.06 or NA 0.07 ) and TSI/DMDS processing.

These initial resolution and linearity results for the TSI process with the DMDS silylation reagent are quite promising. Although the manufacturability of TSI remains a question to be addressed by further experimentation, the improved TSI process using disilanes continues to be a candidate TLI resist technology for EUV lithography. Furthermore, until bilayer or some other TLI process is improved for EUVL, the TSI resist process will continue to be used as an exposure system characterization tool to assess the lithographic performance of EUVL systems that are built or upgraded in the future.

\section{Summary}

While there are several possible TLI resist processes for EUV lithography, the best results to date have been obtained using top surface imaging (TSI) with disilane silylation reagents. The use of disilanes has improved the photospeed, the etch selectivity of the pattern transfer step, and the control of silylated resist flow. The disilane DMDS also eliminated the need for a crosslinker additive to the silylation gas, thereby simplifying the disilane TSI process considerably. Resolution of $100 \mathrm{~nm}$ (equal lines and spaces), linearity down to $100 \mathrm{~nm}$ lines, photospeed of $13 \mathrm{~mJ} / \mathrm{cm}^{2}$, and line edge roughness of $12 \mathrm{~nm}(3 \sigma)$ have all been simultaneously demonstrated for the DMDS silylation process and EUV exposure (NA 0.07). The TSI process will continue to be used as an effective exposure system characterization tool, and improvements in TSI materials and processes are actively being sought as the evaluation of TSI as a manufacturable EUVL resist technology proceeds.

\section{Acknowledgments}

The authors would like to thank several colleagues for their technical contributions to this paper, including Avi Ray-Chaudhuri, Dan Folk, and Kurt Berger at Sandia National Labs, John Hutchinson, Pei-Yang Yan, and Ben de Los Reyes at Intel Corporation, Khanh Nguyen at Advanced Micro Devices, and Scott Hector at Motorola. This work was performed at Sandia National Laboratories, a multiprogram laboratory operated by Sandia Corporation, a Lockheed Martin company, for the U.S. Department of Energy under contract DE-AC04-94AL85000, with funding provided by the Extreme Ultraviolet Limited Liability Company (EUV-LLC).

\section{References}

1. C. G. Willson in Introduction to Microlithography, L.F. Thompson, C. G. Willson, and M. J. Bowden, eds., 2nd edition, American Chemical Society, Washington, D.C. (1994), 239251.

2. E. Reichmanis in Polymers for Electronic and Photonis Applications, C. P. Wong, ed., Academic Press, Inc., San Diego (1993), 67-119.

3. G. N. Taylor, L. E. Stillwagon, and T. Venkatesan, J. Electrochem. Soc. 131 (1984), 1658. 4 T. M. Wolf, G. N. Taylor, T. Venkatesan, and R. T. Kretsch, J. Electrochem. Soc. 131 (1984), 1664. 5. M. A. Hartney, M. Rothschild, R. R. Kunz, D. J. Ehrlich, and D. C. Shaver, J. Vac. Sci. Technol. B 8 (1990), 1476.

6. F. Coopmans and B. Roland, Solid State Technol. 30(6) (1987), 93.

7. S. A. MacDonald, H. Ito, H. Hiraoka, and G. C. Willson, Proc. Reg. Tech. Conf. on Photopolymers, 
Prin., Proc. and Mat., Mid-Hudson Section SPE, Ellenville, New York (1985), 87.

8. C. G. Willson, S. A. MacDonald, H. Ito, J. M. J. Fréchet, Polymers for Microlithography, Kodansha, Tokoyo (1990), 3-18.

9. R. Sezi, R. Leuschner, M. Sebald, H. Ahne, S. Birkle, and H. Börndorfer, Microelectronic Engng. 11 (1990), 535.

10. W. M. Mansfield, O. R. Wood II, R. D. D'Souza, and A. Neureuther, OSA Proceedings Series 12 (1991), 129.

11 D. R. Wheeler, G. Kubiak, A. Ray-Chaudhuri, and C. Henderson, OSA TOPS on Extreme Ultraviolet Lithography 4 (1996), 33.
12. G. N. Taylor, R. S. Hutton, D. L. Windt, and W. M. Mansfield, SPIE Proceedings 1343 (1990), 258. 13. C. Henderson, D. Wheeler, T. Pollagi, D. O'Connell, J. Goldsmith, A. Fisher, G. Cardinale, J. Hutchinson, and V. Rao, SPIE Proceedings 3331 (1998) to be published.

7. R. S. Hutton, G. N. Taylor, and D. R. Wheeler, U.S. patent 5,487,967.

8. M. Irmscher, B. Hofflinger, R. Springer, C. Stauffer, and W. Peterson, J. Photopolym. Sci. Tech. 9(3) (1996), 497. 